\title{
What do weak magnetic fields mean for magnetospheric accretion in Herbig AeBe star+disk systems?
}

\author{
A. N. Aarnio ${ }^{1}$, J. D. Monnier ${ }^{1}$, T. J. Harries ${ }^{2}$ and D. M. Acreman ${ }^{2}$ \\ ${ }^{1}$ Dept. of Astronomy, University of Michigan, 830 Dennison Building, 500 Church Street, Ann \\ Arbor, MI, 48109, USA \\ email: aarnio@umich.edu \\ ${ }^{2}$ School of Physics, University of Exeter, Stocker Road, Exeter, EX4 4QL, UK
}

\begin{abstract}
In the presently favored picture of star formation, mass is transferred from disk to star via magnetospheric accretion and out of the system via magnetically driven outflows. This magnetically mediated mass flux is a fundamental process upon which the evolution of the star, disk, and forming planetary system depends. Our current understanding of these processes is heavily rooted in young solar analogs, T Tauri Stars (TTS). We have come to understand recently, however, that the higher mass pre-main sequence (PMS) Herbig AeBe (HAeBe) stars have dramatically weaker dipolar fields than their lower mass counterparts. We present our current observational and theoretical efforts to characterize magnetospherically mediated mass transfer within HAeBe star+disk systems. We have gathered a rich spectroscopic and interferometric data set for several dozen HAeBe stars in order to measure accretion and mass loss rates, assess wind and magnetospheric accretion properties, and determine how spectral lines and interferometric visibilities are diagnostic of these processes. For some targets, we have observed spectral line variability and will discuss ongoing time-series spectroscopic efforts.
\end{abstract}

Keywords. stars: emission line, Be, stars: evolution, stars: winds, outflows, stars: planetary systems: protoplanetary disks

\section{Introduction}

HAeBe stars are identified as the higher mass pre-main sequence counterparts to TTS (Herbig, 1960). HAeBe stars are observed to have circumstellar disks which, as with the evolution of higher mass stars in generally, dissipate faster than the TTS case (Hernández, 2005). Excess UV luminosity from accretion shocks Calvet \& Gullbring (1998) is observed, as well as P-Cygni absorption features from powerful winds. While radiative transfer modeling has been able to broadly determine physical parameters necessary for a magnetospheric accretion scenario to produce the observed spectral line profiles (Kurosawa, Harries, \& Symington, 2006), the relative contributions of wind and magnetosphere to emission lines remain poorly constrained.

Fundamentally, as developed for TTS, the magnetospheric accretion model depends on a strong, highly ordered dipolar field capable of meeting circumstellar disk material a few stellar radii from the star, channeling it to the stellar surface via accretion streams. Indeed, sufficiently strong fields are observed on TTS (e.g., Basri et al. 1992, JohnsKrull et al. 1999, Johns-Krull, 2007, to name but a few studies), $\sim 1 \mathrm{kG}$ as estimated to be sufficient (Königl, 1991). Recent spectropolarimetric studies of magnetic fields for a range of pre-main sequence stars have shown that the polar dipole strength decreases dramatically with stellar mass (cf. Gregory et al. 2012); this is likely due to the shift from a convective dynamo to a shear-based dynamo (Tout \& Pringle, 1995). As the dipolar 


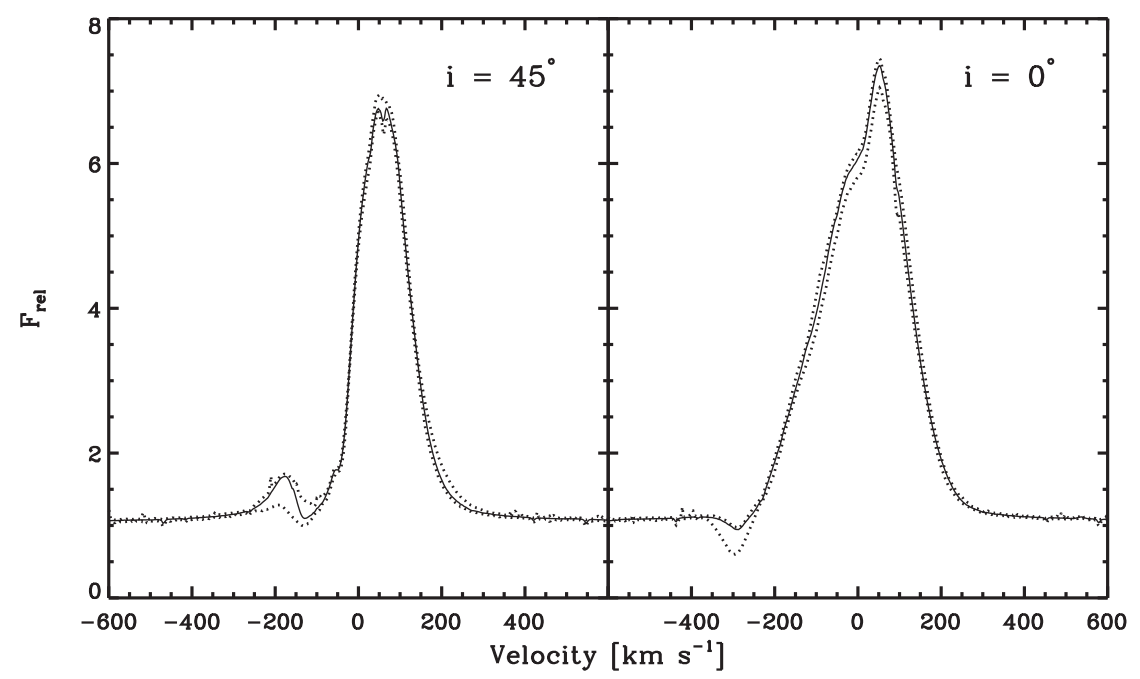

Figure 1. Our time-series optical spectra were taken for a Herbig Be star (spectral type B9, $45^{\circ}$ inclination; left panel) and a Herbig Ae star (A2 spectral type, face-on inclination; right panel). Here we show the dramatic variation observed in the $\mathrm{H} \alpha$ line profiles over the course of 5 days' observation. Solid lines denote the median line profile, dashed lines show the minimum/maximum. Within the nightly observations, we observed strong variability: this underscores the need for simultaneity when comparing spectral lines.

component weakens with increasing stellar mass, higher order moments begin to dominate; in some cases, weak fields are detected on a handful of HAeBe stars (e.g., Hubrig et al. 2009), but a startling number of HAeBe stars have no detected field at all: Alecian et al. (2013) report $\sim 90 \%$ non-detections of magnetic signatures in a spectropolarimetric survey of $70 \mathrm{HAeBe}$ stars. In light of these observations, we ask how, then, are outflows and accretion, magnetically-driven phenomena, occurring on HAeBe stars?

\section{Our HAeBe Observational/Theoretical Campaign}

To address how accretion and outflow happen in HAeBe systems in the limit of weak (or absent) magnetic fields, we are conducting a combined observational and theoretical effort to characterize mass transfer within HAeBe star+disk systems.

\subsection{Spectroscopy and Interferometry}

We have obtained high resolution optical spectra of $\sim 60$ HAeBe stars. These data were taken using the MIKE (Magellan Inamori Kyocera Echelle) spectrograph; MIKE is a dou-

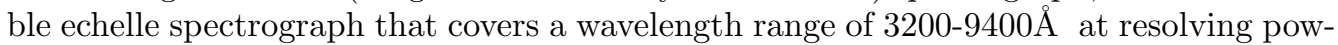
ers $\mathrm{R} \sim 65,000-83,000$. For almost $90 \%$ of these stars, we also have interferometric data, and for another $\sim 70 \%$ we have contemporaneous near-infrared spectra. Additionally, there exists a wealth of photometric data in the literature, which we have used to model spectral energy distributions (SEDs). For some of these targets, we have multi-epoch MIKE spectra, and for two objects, we followed up with time-series optical spectroscopy (Sec. 2.2). The optical data will be presented in a forthcoming publication (Aarnio et al. 2013, in prep), and here we present early results from that sample as well as the time series spectroscopic campaign.

Categorizing the HAeBe $\mathrm{H} \alpha$ profiles using the Reipurth, Pedrosa, \& Lago (1996) morphologies, we find that the vast majority of the sample $(\sim 80 \%)$ shows simple, emissiononly profiles centered at the stellar rest velocity or type-B morphologies with blueshifted 
absorption components in addition to emission components. The remaining line profiles were either II- or III-R; interestingly, in no cases did we see inverse P-Cygni profiles. The type-R line profiles occurred in objects with published high inclination angles.

\subsection{Time Series Spectroscopy}

For two targets that were observed to vary over multiple epochs, we conducted a pilot study, obtaining high cadence time series optical spectroscopy with MIKE to monitor the timescales of emission line variability. In order to assess short-term variability (potentially due to inhomogeneous mass loss or accretion), we observed each object for an hour at the highest possible cadence, $\sim 6 \mathrm{~min}$ and $\sim 4.5 \mathrm{~min}$ with the blue and red sides of MIKE, respectively. To search for variability on the order of the stellar rotation period (e.g., probing asymmetric geometries in the field/accretion streams; Long et al. 2012), we repeated these high-cadence "bursts" for 5 nights within the span of a week. Our final data set consists of $\sim 100$ spectra per object. In Fig. 1, we show the $\mathrm{H} \alpha$ profiles (minus the stellar photospheric component) of these two objects; in order to compare inclination and spectral type effects, we chose one Ae and one Be star, and the stars are face-on and inclined at $45^{\circ}$, respectively. In addition to seeing strong variability on very short timescales in blueshifted absorption features generally attributed to the wind, we also see variability in forbidden lines.

Perhaps surprisingly, we see little variability in the emission components. If the emission originates in a magnetospheric accretion scenario, does a lack of variability imply axisymmetric, steady accretion? Given the unlikeliness of symmetry and steady-state behavior, is the emission then not magnetospheric in origin? Is there some emission from the magnetosphere, but it contributes less than another source? Or, given what we're beginning to understand about magnetism across the H-R diagram (Gregory et al. 2012), is emission from multiple accretion stream components averaging out, making shorter timescale variability undetectable?

\subsection{Line Profile Modeling}

We are in the process of modeling spectral line profiles using TORUS (Transfer of Radiation Under Sobolev; Harries, 2000). For a set of parameters describing the star, disk, disk wind, and magnetosphere, TORUS can produce dust continuum images, SEDs, and atomic line transfer calculations (Fig. 2). Each of these three outputs can then be compared to the interferometric data, literature photometry, and spectroscopy. TORUS is a flexible, modular code which will allow us to modify separate system components individually, updating the disk wind model with information from recent observations, and basing the magnetospheric accretion configuration on extrapolated magnetic field maps from recent observational efforts. We have begun to test some novel magnetic field configurations, and will base these on field extrapolations from recent spectrointerferometric results. Finally, we will take these models and synthesize line profile evolution over a stellar rotation period to compare to time series spectra.

\section{Discussion}

Observationally and theoretically, magnetospheric accretion as it is presently envisaged for TTS has been well established. While it has been generally believed that this paradigm could be extended to HAeBe stars, recent advances in our understanding of stellar magnetic fields as a function of stellar mass have challenged this. Empirical calibrations of spectroscopic indicators and accretion rate, long used for TTS, have been found to break down at higher masses (Mendigutía et al. 2012). 

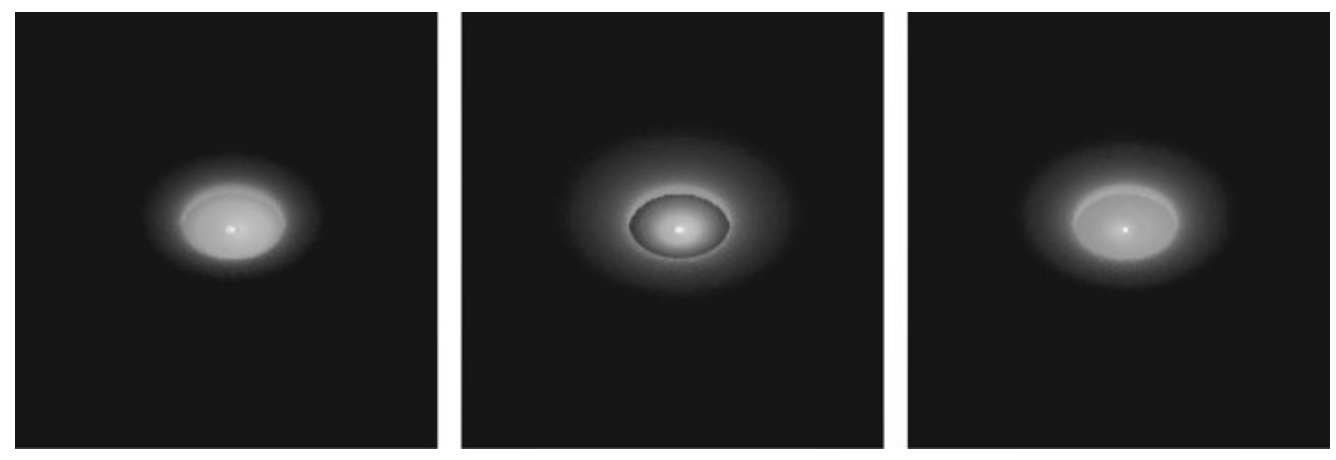

Figure 2. Example TORUS line transfer calculations: $\mathrm{H} \alpha$, for a few magnetospheric configurations. For a fiducial HAeBe star, we show a TTS-like, large-scale magnetosphere that meets the inner gas disk (left), a scaled-down magnetosphere, the dipolar component only extending to $\sim 1.5$ stellar radii (center), and no magnetosphere at all (right). All three models produce strong, centrally peaked $\mathrm{H} \alpha$ emission.

Our combined spectroscopic and interferometric data set allows us to approach the issues of accretion and outflow in HAeBe systems from a unique perspective: simultaneously, we will model line profiles across multiple species, using interferometry to break inclinations in system inclination and disk size which have plagued earlier efforts. We have also seen that looking into the time domain could prove to be a powerful probe of system dynamics, revealing processes occurring on many dynamical timescales and their relationships to one another.

\section{References}

Alecian, E., Wade, G. A., Catala, C., Grunhut, J. H., Landstreet, J. D., Bagnulo, S., Böhm, T., Folsom, C. P., Marsden, S., \& Waite, I. 2013, MNRAS, 429, 1001.

Basri, G., Marcy, G. W., \& Valenti, J. A., 1992, ApJ, 390, 622.

Calvet, N. \& Gullbring, E., 1998, ApJ, 509, 802.

Gregory, S. G., Donati, J.-F., Morin, J., Hussain, G. A. J., Mayne, N. J., Hillenbrand, L. A., \& Jardine, M., 2012, ApJ, 755, 97.

Harries, T. J., 2000, MNRAS, 315, 722.

Herbig, G. H., 1960, ApJS, 4, 337.

Hernández, J., Calvet, N., Hartmann, L., Briceño, C., Sicilia-Aguilar, A., \& Berlind, P., 2005, $A J, 129,856$.

Hubrig, S., Stelzer, B., Schöller, M., Grady, C., Schütz, O., Pogodin, M. A., Curé, M., Hamaguchi, K., \& Yudin, R. V., 2009, A\&̛A, 502, 283.

Johns-Krull, C. M., Valenti, J. A., \& Koresko, C., 1999, ApJ, 516, 900.

Johns-Krull, C. M., 2007, ApJ, 664, 975.

Königl, A., 1991, ApJL, 370, L39.

Kurosawa, R., Harries, T. J., \& Symington, N. H., 2006, MNRAS, 370, 580.

Long, M., Romanova, M. M., \& Lamb, F. K., 2012, New Astronomy, 17, 232.

Mendigutía, I., Mora, A., Montesinos, B., Eiroa, C., Meeus, G., Merín, B., \& Oudmaijer, R. D. 2012, A 6 A 543, A59.

Reipurth, B., Pedrosa, A., \& Lago, M. T. V. T. 1996, A\&SAS, 120, 229.

Tout, C. A. \& Pringle, J. E., 1995, MNRAS, 272, 528. 